\title{
ON CHARACTERS OF HEIGHT ZERO
}

\author{
B. G. BASMAJI \\ (Received 1 December 1980) \\ Communicated by D. E. Taylor
}

\begin{abstract}
Every irreducible ordinary character in a p-block of a finite metabelian group is of height 0 if and only if the defect group of the $p$-block is abelian.

1980 Mathematics subject classification (Amer. Math. Soc.): 20 C 15, $20 \mathrm{C} 20$.

Keywords: characters, representations, modular representations, $p$-blocks, defect groups, height of characters.

Brauer conjectured that all ordinary characters in a $p$-block $B$ of a finite group $G$ have height 0 if and only if the defect group of $B$ is abelian. Fong in [2], [3], [4], and [5] has given proofs of various cases of this conjecture. In this note we prove this for the metabelian groups.
\end{abstract}

THEOREM. Let $G$ be a finite metabelian group and $B$ be a p-block of $G$. Then every ordinary character of $B$ has height 0 if and only if the defect group of $B$ is abelian.

Proof. We use the results in [1]. Let $Q$ be the $p$-Sylow subgroup of the commutator group $G^{\prime}$, then $G^{\prime}=Q \times A$, where $p \nmid|A|$. Let $H$ be a subgroup of $G^{\prime}, H \supseteq Q$, such that $G^{\prime} / H$ is cyclic. Then $H=Q \times \Lambda, p \nmid|\Lambda|$. For any subgroup $L$ of $G^{\prime}$ let $K(L) \supseteq G^{\prime}$, and $K(L) / L$ be a maximal abelian subgroup of $N(L) / L$. If $\Lambda \subseteq L \subseteq H$, we may pick $K(\Lambda) \subseteq K(L) \subseteq K(H)$. Let $\sigma$ be a linear modular representation of $K(\Lambda)$ with ker $\sigma \cap G^{\prime}=H$ and $B(\sigma, H)$ be the collection of all ordinary representations $T^{\prime G}$ where $T^{\prime}$ is a linear representation of $K(L) \supseteq K(\Lambda), L \subseteq H, G^{\prime} / L$ cyclic, $H / L$ a $p$-group, with ker $T^{\prime} \cap G^{\prime}=L$

(1) Copyright Austrialian Mathematical Society 1981 
and $\bar{T}_{K(\Lambda)}^{\prime} G$-conjugate to $\sigma$. See $[1, \S 2]$. All these representations $T^{\prime G}$ are irreducible. Include in $B(\sigma, H)$ the characters of $T^{\prime G}$ and the irreducible composition factors of $\bar{T}^{\prime G}$. From $[1, \S 4], B(\sigma, H)$ is a $p$-block and the $p$-Sylow subgroup $P$ of $K(H)$ is its defect group. [Any $p$-block of $G$ is given by $B(\sigma, H), \sigma$ and $H$ as described above.] Note that $P \cap G^{\prime}=Q$.

First assume $P$ is abelian and let $\pi \in P$. Although this follows from the results in [3] and [4], we give below an easy proof for the special case. Since $K(H) / H$ is abelian, $\pi^{-1} k \pi \equiv k(\bmod H)$ for all $k \in A$. But $\Lambda=H \cap A$, and hence $\pi^{-1} k \pi \equiv k(\bmod \Lambda)$ for all $k \in A$. Since $\pi^{-1} k \pi=k$ for all $k \in Q$, it follows that $\pi^{-1} k \pi \equiv k(\bmod \Lambda)$ for all $k \in G^{\prime}$. Thus $P \subseteq K(\Lambda)$ and $p \nmid$ $|K(H) / K(\Lambda)|$. Since every (irreducible) representation $T^{\prime} G$ in $B(\sigma, H)$ is induced by a linear representation $T^{\prime}$ of $K(L) \supseteq K(\Lambda)$, of some $L$, it follows that the degree of $T^{\prime G}$ divides $\left.|G / K| \Lambda\right) \mid$ but is divisible by $|G / K(H)|$. Thus every ordinary character in $B(\sigma, H)$ has height 0 .

Now assume $P$ is non-abelian. We shall construct an irreducible character in $B(\sigma, H)$ of height greater than 0 . Let $R=P \cap K(\Lambda)$. If $k \in K(\Lambda), \pi \in R$, then $k^{-1} \pi k \equiv \pi(\bmod \Lambda)$. But $\Lambda \cap R=1$, and thus $k^{-1} \pi k=\pi$ for all $\pi \in R$ and all $k \in K(\Lambda)$. Thus $P$ is not contained in $K(\Lambda)$, that is, $R \subset P, R$ abelian, and $K(\Lambda)=R \times K_{1}, p \nmid\left|K_{1}\right|$, with $\Lambda \subseteq K_{1}, K_{1} / \Lambda$ abelian. There is a linear ordinary representation $V$ of $K(\Lambda), V(\pi)=1$ for all $\pi \in R$ and $\bar{V}=\sigma$, ker $V=\operatorname{ker} \sigma$. Since $1 \neq P^{\prime} \subseteq R$ there is a linear ordinary representation $W_{0}$ of $P^{\prime}$, ker $W_{0}=$ $L_{0}$ and $\left|P^{\prime} / L_{0}\right|>1$. Since $R / L_{0}$ is abelian, an extension $W_{1}$ of $W_{0}$ to $R$ exists. Here ker $W_{1} \cap P^{\prime}=L_{0}$. Define the linear representation $W$ of $K(\Lambda)$ by $W(\pi)$ $=W_{1}(\pi)$ for all $\pi \in R$ and $W(k)=1$ for all $k \in K_{1}$. Let $T(k)=V(k) W(k)$ for all $k \in K(\Lambda)$. Then $T$ is a linear representation of $K(\Lambda)$ and $\bar{T}=\sigma$. Let $L=\operatorname{ker} T \cap G^{\prime}$ and $K(L) \supseteq K(\Lambda)$. Then $L \cap P^{\prime}=L_{0}$ and thus there are $\pi_{1} \in P^{\prime}$ and $\pi \in P$ such that $\pi^{-1} \pi_{1} \pi \not \pi_{1}\left(\bmod L_{0}\right)$. This means that $\pi^{-1} \pi_{1} \pi \not$ $\pi_{1}(\bmod L)$ or $P$ is not contained in $K(L)$. Let $T^{\prime}$ be an extension of $T$ to $K(L)$, then $T^{\prime G}$ is irreducible and since $\bar{T}_{K(\Lambda)}^{\prime}=\sigma, T^{\prime G} \in B(\sigma, H)$. Now since $T^{\prime G}$ is of degree $|G / K(L)|$ and $p|| K(H) / K(L) \mid$, it follows that its character $\chi$ is of positive height. This completes the proof of the theorem.

\section{References}

[1] B. G. Basmaji, 'Modular representation of metabelian groups', Trans. Amer. Math. Soc. 169 (1972), 389-399. MR 46 \#9153.

[2] P. Fong, 'Some properties of characters of finite solvable groups', Bull. Amer. Math. Soc. 66 (1960), 116-117. MR 22 \#2655.

[3] P. Fong, 'On the characters of p-solvable groups', Trans. Amer. Math. Soc. 98 (1961), 263-284. MR 22 \#11052. 
[4] P. Fong, 'Solvable groups and modular representation theory', Trans. Amer. Math. Soc. 103 (1962), 484-494. MR 25 \#3098.

[5] P. Fong, 'A note on a conjecture of Brauer', Nagoya Math. J. 22 (1963), 1-13. MR 27 \#3703.

Mathematics and Computer Science Department

California State University

Los Angeles, California 90032

U.S.A. 